\title{
A Comparative Study of Right Ventricular Apical Pacing and Right Ventricular Septal Pacing
}

\author{
Shimao $\mathrm{Ni}^{*}$, Changhao Jiang and Zhili Chen \\ Department of Cardiology, Yiwu Central Hospital, Yiwu, Zhejiang 322000, China
}

\begin{abstract}
Objective: Assessment of cardiac function by the assessment of ventricular pacing and right ventricular apex pacing. Method: Analysis of 30 patients with VVI pacemaker (pacing RVA, right ventricular apical pacing RVS 15 cases) was analyzed, and the differences of cardiac function and pacing parameters were analyzed in one year. Results: There was no significant difference in the impedance, voltage threshold and $\mathrm{R}$ wave height between the 1 year after the effective pacing and the years after the pacing electrode. 1 year after the two groups of patients with significant differences in EF. Conclusion: Right ventricular septal pacing and right ventricular apical pacing are equally safe and effective, right ventricular apical pacing can lead to left ventricular systolic dysfunction and decreased left ventricular function.
\end{abstract}

\author{
KEYWORDS \\ Right ventricle \\ Ventricular septum \\ Systolic dysfunction
}

\section{Introduction}

Pacemaker has been used in clinical for 56 years as early treatment of patients with slow heart rate. During the period, the majority of scholars have carried out a lot of research, especially in the pacing mode and pacing position. The right ventricular apex is easy to reach, and the fixation is convenient. So far, it is widely accepted, but its influence on the double ventricular electrical synchrony, the cardiac cell degeneration, fibrosis and so on, which leads to the decrease of cardiac function still needs to be changed into physiological sequential pacing mode $[1,2]$. However, Right Ventricular Septal (RVS) pacing is close to the His bundle, to get close to the physiological state of the left and right ventricular synchronization and has little effect on the cardiac function in which can not only take into account the quality of life, but also pay more attention to the long-term impact of the patient's hemodynamics and heart function. In this paper, the effects of Right Ventricular Apical (RVA) pacing and Right Ventricular Septal (RVS) pacing on pacing parameters and cardiac function were

Copyright () 2015 Shimao Ni et al.

doi: $10.18686 /$ aem.v4i1.5

Received: December 3, 2014; Accepted: February 8, 2015; Published online: March 19, 2015

This is an open-access article distributed under the terms of the Creative Commons Attribution Unported License (http://creativecommons.org/ licenses/by-nc/4.0/), which permits unrestricted use, distribution, and reproduction in any medium, provided the original work is properly cited.

${ }^{\star}$ Corresponding author: Department of Cardiology, Yiwu Central Hospital, Yiwu, Zhejiang 322000, China. E-mail: 478215335@qq.com analyzed.

\section{Materials and methods 2.1. General information}

The study planned to include 30 of patients in our hospital who with VVI pacemaker implantation which were randomized into RVS group of 15 cases, including 7 cases of sick sinus syndrome, 8 cases of atrioventricular block and at average age $(62+1.8)$ years old. For RVA group, there were 15 of cases, average age $(62.7+1.2)$ years including 6 cases of sick sinus syndrome and 9 cases of atrioventricular block. Inclusion criteria were as follows: (1) As intermittent and continuous of second degree or third degree atrioventricular block patients; (2) Age around 50 to 74 years old; (3) No patients were combined with hypertension, diabetes and other chronic diseases; (4) Pre-operative ECG without bundle branch and intraventricular block (5) left ventricular ejection fraction $(\mathrm{LVEF})>0.50$.

\subsection{Method}

(1) The electrode implantation method: All the patients will be fixed in the right auricle atrial electrode. Patients were randomized into two groups: group A is Right Ventricle Outflow Tract (RVOT) pacing where was fed by the active spiral electrode and group B is RVA pacing were the passive electrode was fixed in the right ventricular apex. According to the time limit of pacing, pacing threshold, $\mathrm{R}$ wave, QRS wave is adjusted to the best state, and the fixed electrode is fixed. (2) The test of pacing parameters: the MEDTRONIC 5318 type temporary pacemaker was used 
to test the program. (3) Connected and embedded pulse generator (4) Holter results were collected after one week by Medtronic 2090 pacemaker programmer and again program-controlled $\mathrm{A}-\mathrm{V}$ interval (between $120150 \mathrm{~ms}$ ). This is to ensure that each case patient have ventricular average pacing rate above $90 \%$. Determinations of Ejection Fracture (EF) value of the patients in each group after the positioning of the pacing analyze were analyses.

\subsection{Statistical methods}

All statistical analysis of the data was statistically analyzed by SPSS14.0 statistics software. Continuous data were expressed as mean \pm standard deviation $(\bar{x} \pm s)$. Student $t$ test analyses for comparison between groups, all were done as 2 -sided tests. $p<0.05$ was considered statistically significant.

\section{Result}

After one years of implantation, there was no significant difference between the two groups in the pacing threshold, sensing threshold value, and the impedance parameters $(p$ $>0.05)$. The EF values of the RVA group were significantly decreased, and the difference was significant $(p<0.05)$, while the QRS group was significantly higher than that in the RVS group $(p<0.05)$.

\section{Discussion}

Sinoatrial node serves as the natural pacemaker for the heart. It sends the electrical impulse that triggers each heartbeat. The impulse then strikes Atrioventricular node which is located in the lower part of right atrium. Next, electrical stimulus is passes through the Arterioventricular node and Bundle of His, divides into left and right bundle brunches and passes through the Purkinje fiber networks causing both ventriculars to contract. This pushes blood through pulmonary valves to the lungs and the rest of the body [3]. This process continues over and over as atrial and ventricular sequential are excited so as to achieve the physiological processes of body. The simultaneous activation of the two chambers is a powerful guarantee for the maximization of cardiac hemodynamics. Right ventricular apical pacing is a main mechanism of the development of heart failure. In addition, right ventricular apical pacing leads to myocardial perfusion imbalance, myocardial material and energy metabolism disorders. Myocardial cell oxygen consumption and cardiac remodeling are also an important factor affecting the long-term cardiac function. Studies have shown that the right ventricular septal pacing compared with right ventricular apical pacing significantly increased cardiac output and stroke volume. Possible mechanism leading to this advantages are as follow: (1) The pacing electrode near the His bundle, excited by nearby bundle rapid transfer to the Purkinje's network, causing physiological biventricular synchronous excitation activity which retains the maximum effect of hemodynamics; (2) Able to reduce activation of neurohormones cytokine and reduce myocardial oxygen consumption able to avoid myocardial substance and energy metabolism disorders, from the pathology of science to prevent myocardial remodeling [4]; (3) Biventricular coordinated motion were balanced, and able to block the long-term ventricular wall stage of contradiction movement and function of mitral valve.

Commonly, cardiac pacemaker implantation has been implanted to the right ventricular apex. This is because the position of the muscle is small, easy to fix, and the operation is simple. The study showed that the incidence of heart failure in the right ventricular apical pacing patients with QRS wave duration was increased where the main caused was decreased the left ventricular systolic ventricular filling and the decrease of the amount of blood $[5,6]$. RVS pacing using cardiac conduction system of the heart muscle tissue can maintain the right and left ventricular (LV) in normal order and synchronous contraction. Cardiac output volume has been significantly improved (average increase of about $12.24 \%$ ) of the ECG and QRS time relative to RVA. Many years of clinical research and demonstration have found that the RVA pacing has been used as a result RVA can reduce the defects caused by the traditional pacing.

In summary, the myocardial contraction caused by RVS pacing is more in line with the physiological and clinical practice. The theory is significantly better in RVS than in RVA pacing. Therefore, there is no objection to block the need for pacemaker patients whereby right ventricular septal pacing is safe, reliable and more ideal pacing mode. As a conclusion, RVS is better than right ventricular apex pacing and is worthy for clinical application.

\section{Conflicts of interest}

These authors have no conflicts of interest to declare.

\section{Authors' contributions}

These authors contributed equally to this work.

Table 1. Pacing parameters in two groups of patients $(\bar{x} \pm s)$.

\begin{tabular}{|c|c|c|c|c|c|c|c|}
\hline & \multirow{3}{*}{ Group } & \multirow{3}{*}{$\begin{array}{c}\text { Cases Threshold } \\
(\mathrm{mV})\end{array}$} & \multirow{3}{*}{$\begin{array}{l}\text { Pacing Threshold } \\
\text { (V) }\end{array}$} & \multirow{3}{*}{$\begin{array}{c}\text { Perception } \\
\text { impedance }(\Omega)\end{array}$} & \multirow{3}{*}{$\begin{array}{l}\text { Electrode } \\
\quad(\mathrm{ms})\end{array}$} & \multicolumn{2}{|c|}{ Pacing QRS } \\
\hline & & & & & & \multicolumn{2}{|c|}{ EF value (\%) } \\
\hline & & & & & & Pre-operative & Post-operative \\
\hline RVA & 15 & $0.42 \pm 0.13$ & $14.1 \pm 1.4$ & $875 \pm 46$ & $132 \pm 11.6$ & $66.1 \pm 3.3$ & $41.5 \pm 6.8$ \\
\hline RVS & 15 & $0.54 \pm 0.12^{* *}$ & $13.3 \pm 2.1^{* *}$ & $902 \pm 39^{* *}$ & $105.3 \pm 2.4^{*}$ & $64.8 \pm 5.1^{*}$ & $62.7 \pm 8.2^{*}$ \\
\hline
\end{tabular}

Note: compared with RVA group, $p<0.05^{*}, p>0.05^{* *}$. 


\section{References}

1. Lamas GA, Ellenbogen DA, Hennekens $\mathrm{CH}$, et al. Evidence base for pacemakermode selection from physiology to randomized trials. Circulation. 2004;109(4):443451.

2. Cock CC, Giudici MC, Twisk JW. Comparison of the haemodynamic effects of right ventricular outflow tract pacing with right ventricular apex pacing. Europace. 2003;5(3):275-278.

3. Drazner MH, Thompson B, Rosenberg PB, et al. Comparison of impedance cardiography with invasive hemodynamic measurements in patients with heart failure secondary to ischemic or nonischemic cardiomyopathy. Am J
Cardiol. 2002;89(8):993-995.

4. Shukla HH, Hellkamp AS, James EA, et al. Heart failure hospitalization is more common in pacemaker patients with sinus node dysfunction and a prolonged paced QRS duration. Heart Rhythm. 2005;2(3):252-256

5. Miyoshi $\mathrm{F}$, Kobayashi $\mathrm{Y}$, Itou $\mathrm{H}$, et al. Prolonged paced QRS duration as a predictor for congestive heart failure in patients with right ventricular apical pacing. PACE. 2005;28(11):1182-1186.

6. Rosenqvist M, Lsaaz K, Botvinick EH, et al. Relative importance of activation sequence compared to a atrioventricular synchrony in left ventricular function. Am J Cardiol. 1991;67(2):148-156. 